\title{
Flux-cutting and flux-transport effects in type-II superconductor slabs in a parallel rotating magnetic field
}

\author{
R. Cortés-Maldonado ${ }^{1}$, J.E. Espinosa-Rosales ${ }^{2}$, A.F. Carballo-Sánchez ${ }^{3}$, and F. Pérez-Rodríguez ${ }^{* 1}$ \\ ${ }^{1}$ Instituto de Física, Benemérita Universidad Autónoma de Puebla, \\ Apdo. Post. J-48, Puebla, Pue. 72570, Mexico \\ ${ }^{2}$ Facultad de Ciencias Físico-Matemáticas, Benemérita Universidad Autónoma de Puebla, \\ Apdo. Post. 1152, Puebla, Pue., 72000, Mexico \\ ${ }^{3}$ Universidad del Istmo, Campus Tehuantepec, Tehuantepec, Oax., 70760, Mexico
}

\begin{abstract}
The magnetic response of irreversible type-II superconductor slabs subjected to in-plane rotating magnetic field is investigated by applying the circular, elliptic, extended-elliptic, and rectangular flux-line-cutting critical-state models. Specifically, the models have been applied to explain experiments on a $\mathrm{PbBi}$ rotating disk in a fixed magnetic field $\boldsymbol{H}_{a}$, parallel to the flat surfaces. Here, we have exploited the equivalency of the experimental situation with that of a fixed disk under the action of a parallel magnetic field, rotating in the opposite sense. The effect of both the magnitude $H_{a}$ of the applied magnetic field and its angle of rotation $\alpha_{s}$ upon the magnetization of the superconductor sample is analyzed. When $H_{a}$ is smaller than the penetration field $H_{P}$, the magnetization components, parallel and perpendicular to $\boldsymbol{H}_{a}$, oscillate with increasing the rotation angle. On the other hand, if the magnitude of the applied field, $H_{a}$, is larger than $H_{P}$, both magnetization components become constant functions of $\alpha_{s}$ at large rotation angles. The evolution of the magnetic induction profiles inside the superconductor is also studied.
\end{abstract}

PACS numbers: 74.25.Ha, 74.25.Op, 74.25.Sv, 74.25.Wx

Keywords: flux cutting, flux transport, vortex pinning, critical state, hard superconductor

\section{INTRODUCTION}

The discovery of the phenomenon known as quasisymmetrical collapse of magnetization 1, which is observed in superconductors subjected to crossed magnetic fields and well interpreted within the simple Bean's critical-state model [2, 3], has been a turning point in the understanding of the magnetic behavior of hard (irreversible type-II) superconductors. Until then, the generalized double critical-state model (GDCSM) 4, 5, 6, 7, 8, which is based on fundamental physical concepts such as flux transport and fluxline-cutting [9, 10], was successfully employed to explain a variety of experiments where flux cutting occurs 11, 12, 13, 14, 15, 16]. An important feature of the GDCSM is the assumption that flux cutting and flux depinning do not affect each other. Besides, the GDCSM is inherently anisotropic because the thresholds for these two effects are given by two independent parameters, namely the critical current densities parallel $\left(J_{c \|}\right)$ and perpendicular $\left(J_{c \perp}\right)$ to the local magnetic induction $\boldsymbol{B}$. However, since the GDCSM cannot reproduce the features of magnetic moment collapse [17, 18], whereas isotropic Bean's model does it, the main assumption of the GDCSM has been questioned, motivating the development of new critical-state mod- els in the past few years.

In Ref. [19, the so-called elliptic flux-line-cutting critical-state model was proposed. This model introduces the anisotropy, induced by flux-line-cutting effects, by using a procedure similar to that for structurally anisotropic superconductors [20, 21], i.e. the magnitude of the critical current density $J_{c}$, being the only parameter used within the isotropic Bean's model, is substituted by a symmetrical tensor $\left(J_{c}\right)_{i k}$ with principal values $J_{c \|}$ and $J_{c \perp}$, corresponding to the directions along and across the local magnetic induction $\boldsymbol{B}$. In good agreement with the experiment on $\mathrm{YBa}_{2} \mathrm{Cu}_{3} \mathrm{O}_{7-\delta}$ samples [1, 17, the elliptic criticalstate model predicts the quasisymmetrical suppression of the average magnetization $\left\langle M_{z}\right\rangle$, for paramagnetic and diamagnetic initial states, by sweeping a transverse field $H_{y}$ of magnitude much smaller than dc-bias magnetic field $H_{z}$ [19, 22. When the magnitudes of the crossed fields $H_{y}$ and $H_{z}$ are comparable, the value of the magnetization $\left\langle M_{z}\right\rangle$ after many cycles of the transverse field $H_{y}$ turns out to be positive for both diamagnetic and paramagnetic initial states if $J_{c \|}>J_{c \perp}$. To our knowledge, the observation of such a paramagnetism of hard superconductors was first reported in Refs. [23, 24]. The elliptic model also describes the behavior of $\left\langle M_{y}\right\rangle\left(H_{y}\right)$

\footnotetext{
*Author to whom correspondence should be addressed: fperez@ifuap.buap.mx
} 
and $\left\langle M_{z}\right\rangle\left(H_{y}\right)$ in crossed fields $H_{y}$ and $H_{z}$ [19, 25], which was observed in the experiments on a VTi ribbon with nonmagnetic initial state [14, 26. Here, the good agreement with the experiment was achieved by using a relatively large anisotropy parameter $J_{c \|} / J_{c \perp}=6$. It should be noticed that the Bean's critical state model predicts neither the phenomenon of the paramagnetism of hard superconductors nor the behavior of the components of the average magnetization found in Refs. [14, 26]. Furthermore, as it is shown in Refs. [19, 27], the elliptic critical-state model successfully describes the magnetic response of superconducting disks undergoing oscillations in a magnetic field of fixed magnitude for nonmagnetic, paramagnetic, and diamagnetic initial states [1].

Despite the great success of the elliptic model [19], it turns out that there exist phenomena, associated with flux cutting, which are not completely described within such a model. So, in a very recent work [28], the elliptic critical-state model and other four theoretical approaches for describing the critical state of type-II superconductors (GDCSM, extended GDCSM [29, 30, extended elliptic critical-state model [28, 31, and an elliptic critical-state model based on the variational principle [32]) were tested. There, the angular dependencies of the critical current density $J_{c}$ and the electric field $\boldsymbol{E}$ (for $J$ just above $J_{c}$ ) were measured, using an epitaxially grown YBCO thin film, and compared with the predictions of the five theories. The measurements of angular dependence of the criticalcurrent density $J_{c}$ demonstrated a behavior rather similar to that assumed by the elliptic critical-state models. Besides, the smooth angular dependence of the ratio of the transverse to the longitudinal components of the electric field $E_{y} / E_{z}$ for $J$ just above $J_{c}$, predicted by the three elliptic models, was verified in the experiment 28]. However, the original critical-state model [19] leads to small values of the ratio $E_{y} / E_{z}$ in comparison with the experimental data and the results obtained from the other two elliptic models. On the basis of this detailed comparison between experiment and the five theories, it was concluded in Ref. [28] that the experiment favors only one of the models, namely the extended elliptic critical-state model.

The aim of the present work is to investigate the behavior of a hard superconductor in a parallel rotating magnetic field (or equivalently, the response of a rotating superconductor in a fixed magnetic field) and to compare the predictions of four critical-state models with experiment. Concretely, we shall consider the Bean's critical-state model [2, 3], the original elliptic critical-state model [19, 22, the recently-proposed extended elliptic model [28, 31, as well as the GDCSM 44, 5, 6, 7, 8, whose main characteristics and assumptions will be revisited in Sec. III. We shall numerically solve Maxwell equations with the material equation postulated by each of the considered critical-state models to calculate magnetization curves for a superconductor disk rotating in a fixed magnetic field as in the experiment [33] (Sec. III.). Here, we shall analyze the effect of the magnitude $H_{a}$ of the applied magnetic field upon the dependencies of the magnetization components, parallel and perpendicular to $\boldsymbol{H}_{a}$, on the rotation angle of the superconductor disk. The evolution of magnetic induction profiles will also be studied to explain the magnetic response of the rotating hardsuperconductor sample.

\section{THEORETICAL FORMALISM}

Let us consider a superconducting slab of thickness $d$, which occupies the space $0<x<d$ and is subjected to a magnetic field $\boldsymbol{H}_{a}$ parallel to its surfaces:

$$
\boldsymbol{H}_{a}=H_{a} \hat{\boldsymbol{\alpha}}_{s}=H_{a}\left[\hat{\boldsymbol{y}} \sin \left(\alpha_{s}\right)+\hat{\boldsymbol{z}} \cos \left(\alpha_{s}\right)\right],
$$

where $\alpha_{s}$ is the angle of the applied magnetic field $\boldsymbol{H}_{a}$ with respect to the $z$-axis. Hence, the magnetic induction $\boldsymbol{B}(x, t)$ inside the superconducting slab can be expressed as

$$
\boldsymbol{B}=B(x, t)[\hat{\boldsymbol{y}} \sin (\alpha(x, t))+\hat{\boldsymbol{z}} \cos (\alpha(x, t))],
$$

where $B$ and $\alpha$ are respectively the magnitude and the tilt angle of the magnetic induction. It is convenient to write the electric field $\boldsymbol{E}(x, t)$ and the electrical current density $\boldsymbol{J}(x, t)$ in terms of their components parallel and perpendicular to the local magnetic induction $\boldsymbol{B}(x, t)$ :

$$
\begin{aligned}
\boldsymbol{E}(x, t) & =E_{\|}(x, t) \hat{\boldsymbol{\alpha}}(x, t)+E_{\perp}(x, t) \hat{\boldsymbol{\beta}}(x, t), \\
\boldsymbol{J}(x, t) & =J_{\|}(x, t) \hat{\boldsymbol{\alpha}}(x, t)+J_{\perp}(x, t) \hat{\boldsymbol{\beta}}(x, t),
\end{aligned}
$$

where $\hat{\boldsymbol{\beta}}(x, t)=\hat{\boldsymbol{x}} \times \hat{\boldsymbol{\alpha}}(x, t)$. Inside the superconductor sample, we shall assume that the magnetic induction and the magnetic field satisfy the relation $\boldsymbol{B}(x, t)=\mu_{0} \boldsymbol{H}(x, t)$, which is good enough for applied magnetic fields much larger than the first critical field $\left(H_{a} \gg H_{c 1}\right)$. Moreover, any surface barrier against the flux entry (or exit) will be neglected. According to the planar geometry of the problem, we can rewrite Ampere and Lorentz laws,

$$
\begin{aligned}
\nabla \times \boldsymbol{B}(x, t) & =\mu_{0} \boldsymbol{J}(x, t), \\
\nabla \times \boldsymbol{E}(x, t) & =-\frac{\partial \boldsymbol{B}}{\partial t},
\end{aligned}
$$

as follow

$$
\begin{gathered}
\frac{\partial B}{\partial x}=-\mu_{0} J_{\perp}, \\
B \frac{\partial \alpha}{\partial x}=-\mu_{0} J_{\|}, \\
\frac{\partial E_{\perp}}{\partial x}+E_{\|} \frac{\partial \alpha}{\partial x}=-\frac{\partial B}{\partial t}, \\
E_{\perp} \frac{\partial \alpha}{\partial x}-\frac{\partial E_{\|}}{\partial x}=-B \frac{\partial \alpha}{\partial t} .
\end{gathered}
$$

To solve the resulting system of differential equations for $\boldsymbol{E}, \boldsymbol{B}$ and $\boldsymbol{J}$, one should add the material equation. Below, we shall use the material equations corresponding to the circular, elliptic, extended-elliptic, and rectangular flux-line-cutting critical-state models. 


\section{A. Circular model}

The first model for describing the magnetic behavior of superconductors in multicomponent situations was proposed by Bean [2, 3]. According to it, the critical current density $\boldsymbol{J}$ points always along the local electric field $\boldsymbol{E}$. Hence,

$$
\boldsymbol{J}=J_{c} \frac{\boldsymbol{E}}{E} .
$$

The magnitude of the critical current density $J=J_{c}$ is the unique phenomenological parameter used and may depend on the magnitude of the magnetic induction $B$. In the planar geometry [see Eqs. 2.1)-(2.4)], the assumption $J=J_{c}$ corresponds to a circle in the $J_{\perp^{-}}$ $J_{\|}$plane.

In numerically solving the system of equations (2.7)-2.10 for the electromagnetic fields, it is necessary to rewrite Eq. 2.11 as

$$
\begin{gathered}
\boldsymbol{E}=E(J) \frac{\boldsymbol{J}}{J} \\
E(J)= \begin{cases}0, & J \leq J_{c}(B) \\
\rho\left(J-J_{c}(B)\right), & J \geq J_{c}(B)\end{cases}
\end{gathered}
$$

where $\rho$ is an effective resistivity. It should be mentioned that for slow variations of the surface boundary conditions, producing a small magnitude of the induced electric field $\left(E \ll \rho J_{c}\right)$, the magnetic induction profiles are practically relaxed and independent of the parameter $\rho$ [34].

\section{B. Elliptic model}

The elliptic flux-line cutting critical-state model [19, 22, 25] postulates:

$$
J_{i}=\left(J_{c}\right)_{i k} \frac{E_{k}}{E}
$$

where

$$
\left(J_{c}\right)_{i k}=J_{c, i}(B) \delta_{i j}, \quad i, k=\perp, \| .
$$

Here $\delta_{i k}$ is the Kronecker delta symbol. Within the elliptic critical-state model (2.14), the magnitude of the critical current density $J_{c}$ draws an ellipse on the $J_{\perp}-J_{\|}$ plane. This model makes use of two phenomenological parameters, namely the extreme values $J_{c \perp}$ and $J_{c \|}$ for the radius of the ellipse drawn by the magnitude of the critical current density. In the numerical calculations for solving the system of equations 2.7)- 2.10), the relation $(2.14)$ is rewritten in the form

$$
\begin{gathered}
E_{i}=E(J)\left(J_{c}^{-1}\right)_{i k} J_{k}, \\
E(J)=\left\{\begin{array}{ll}
0, & J \leq J_{c}(B, \phi) \\
\rho\left(J-J_{c}(B, \phi)\right), & J \geq J_{c}(B, \phi)
\end{array},\right.
\end{gathered}
$$

where $\left(J_{c}^{-1}\right)_{i k}$ is the inverse of the matrix $\left(J_{c}\right)_{i k}$ in (2.14). The magnitude of the critical current density, $J_{c}(B, \phi)$, is given by the expression

$$
J_{c}(B, \phi)=\left[\frac{\cos ^{2}(\phi)}{J_{c \|}^{2}(B)}+\frac{\sin ^{2}(\phi)}{J_{c \perp}^{2}(B)}\right]^{-1 / 2} .
$$

Here, $\phi$ denotes the angle of the critical current density $\boldsymbol{J}$ with respect to the direction of the flux density B. If $J_{c \perp}=J_{c \|}$, the elliptic critical-state model 2.16 goes over into the Bean's (circular) critical-state model 2.12). Besides, the calculations of electromagnetic fields with $J$ close to $J_{c}$ are also independent of the auxiliary parameter $\rho$ in Eq. 2.17).

\section{Extended elliptic model}

The elliptic critical-state model, described in previous subsection, has recently been extended in Refs. 28, 31 by introducing the general relations

$$
\begin{aligned}
E_{\perp} & =\rho_{\perp} J_{\perp}, \\
E_{\|} & =\rho_{\|} J_{\|},
\end{aligned}
$$

where $\rho_{\perp}$ and $\rho_{\|}$are nonlinear effective resistivities, having a ratio $r=\rho_{\|} / \rho_{\perp}$ independent of $J$ just above $J_{c}$ as it was experimentally found [28]. A model for the effective resistivities is given by 31

$$
\begin{gathered}
E_{\perp}=\left\{\begin{array}{ll}
0, & 0 \leq\left|J_{\perp}\right| \leq J_{c d} \\
\rho_{d}\left(\left|J_{\perp}\right|-J_{c d}\right) \operatorname{sign}\left(J_{\perp}\right), & \left|J_{\perp}\right| \geq J_{c d}
\end{array},\right. \\
E_{\|}= \begin{cases}0, & 0 \leq\left|J_{\|}\right| \leq J_{c c} \\
\rho_{c}\left(\left|J_{\|}\right|-J_{c c}\right) \operatorname{sign}\left(J_{\|}\right), & \left|J_{\|}\right| \geq J_{c c}\end{cases}
\end{gathered}
$$

Here, the subscripts " $\mathrm{d}$ " and "c" respectively refer to depinning and cutting. Besides, $J_{c d}=J_{c}(B, \phi)|\sin (\phi)|$ and $J_{c c}=J_{c}(B, \phi)|\cos (\phi)|$, where $J_{c}(B, \phi)$ is defined according to the elliptic critical-state model as in Eq. 2.18. If $\left|J-J_{c}\right| / J_{c} \ll 1$, the extended elliptic critical-state model reduces to the original one [Eqs 2.16 and 2.17) by replacing $\rho_{d}$ and $\rho_{c}$ in Eqs. 2.21 and $\left(2.22\right.$ with $\rho J_{c} / J_{c \perp}$ and $\rho J_{c} / J_{c \|}$, correspondingly. Hence, in the case of the original elliptic model, the ratio $r=\rho_{\|} / \rho_{\perp}$ at $J>J_{c}$ is equal to $J_{c \perp} / J_{c \|}$. On the other hand, the extended elliptic critical-state model is capable to modify the relation between the components of the electric field $\boldsymbol{E}$ and the current density $\boldsymbol{J}$ with the aid of the additional parameter $r$.

\section{Rectangular model}

The generalized double critical-state model (GDCSM) [4, 5, 6, 7, 8] uses two phenomenological parameters, namely the critical values, $J_{c \|}$ and $J_{c \perp}$, of the electrical current density along and perpendicular to the local magnetic induction. Within this model, each component of the electrical current density is determined by its own electric field as

$$
\begin{gathered}
J_{\perp}=J_{c \perp} \operatorname{sign}\left(E_{\perp}\right), \\
J_{\|}=J_{c \|} \operatorname{sign}\left(E_{\|}\right) .
\end{gathered}
$$

Evidently, the magnitude of the critical current density traces a rectangle in the $J_{\perp}-J_{\|}$plane. The parameter $J_{c \perp}$ determines the threshold for depinning of vortices, 
whereas $J_{c \|}$ indicates the onset of flux-line cutting in the vortex array. In calculating the electromagnetic fields within the GDCSM, the material equation 2.23 is written in the form

$$
\begin{gathered}
E_{\perp}=\left\{\begin{array}{ll}
0, & 0 \leq\left|J_{\perp}\right| \leq J_{c \perp} \\
\rho_{\perp}\left(\left|J_{\perp}\right|-J_{c \perp}\right) \operatorname{sign}\left(J_{\perp}\right), & \left|J_{\perp}\right| \geq J_{c \perp}
\end{array},\right. \\
E_{\|}= \begin{cases}0, & 0 \leq\left|J_{\|}\right| \leq J_{c \|} \\
\rho_{\|}\left(\left|J_{\|}\right|-J_{c \|}\right) \operatorname{sign}\left(J_{\|}\right), & \left|J_{\|}\right| \geq J_{c \|}\end{cases}
\end{gathered}
$$

The quantities $\rho_{\perp}$ and $\rho_{\|}$are effective flux-flow and flux-line-cutting resistivities of the material. However, unlike the above-commented critical-state models, the GDCSM allows the existence of zones in the $J_{\perp}-J_{\|}$ plane where either flux cutting or flux transport exclusively occur. The latter is possible due to the assumption of the GDCSM that the threshold for flux depinning, $J_{c \perp}$ (flux cutting, $J_{c \|}$ ) is independent of the component $J_{\|}\left(J_{\perp}\right)$ [compare Eqs. 2.25) and 2.26) with Eqs. 2.21) and (2.22) where $J_{c d}$ and $J_{c c}$ depend on the angle $\left.\phi=\arctan \left(J_{\perp} / J_{\|}\right)\right]$.

\section{NUMERICAL RESULTS AND COMPARISON WITH EXPERIMENT}

In the present section we will apply the flux-linecutting critical-state models, commented above, to explain experimental magnetization curves 33 of a $\mathrm{PbBi}$ superconducting disk, rotating in the presence of an external magnetic field $\boldsymbol{H}_{a}$, which is oriented parallel to the disk plane (along the $z$-axis) and perpendicular to the axis of rotation.

\section{A. Experimental results}

Fig. 11a exhibits a standard magnetization curve, which was measured in Ref. [33], for a PbBi disk of thickness $d=0.8 \mathrm{~mm}$. The hysteresis in Fig. 1 a clearly corresponds to the magnetization curve of a type-II irreversible superconductor since its return crosses over and remains in the paramagnetic region as a result of the strong flux pinning. In the experiment, the isotropy of the PbBi disk was also verified by comparing standard magnetization curves with $\boldsymbol{H}_{a}$ directed along different diameters of the disk.

Panels (a)-(c) in Fig. 2 show graphs of the magnetization components, $<M_{y}>=<B_{y}>/ \mu_{0}$ and $\left.-<M_{z}\right\rangle=H_{a}-\left\langle B_{z}>/ \mu_{0}\right.$, versus the angle $\theta$ of rotation, measured in the work 33 for the $\mathrm{PbBi}$ disk, rotating in the magnetic field $\boldsymbol{H}_{a}$. The measurements started in the nonmagnetic initial state which is reached after cooling the superconductor at the fields $H_{a} / H_{P}=0.5$ (panel a), 1.0 (panel b), and 2.0 (panel c), where $H_{P}\left(\mu_{0} H_{P}=0.1015 \mathrm{~T}\right.$ [33] $)$ is the penetration field. The initial state is supposed to be nonmagnetic because no Meissner effect (flux expulsion) was observed after field cooling, within the accuracy ( $\Delta<M>\leq 1$ Gauss) of the experiment.
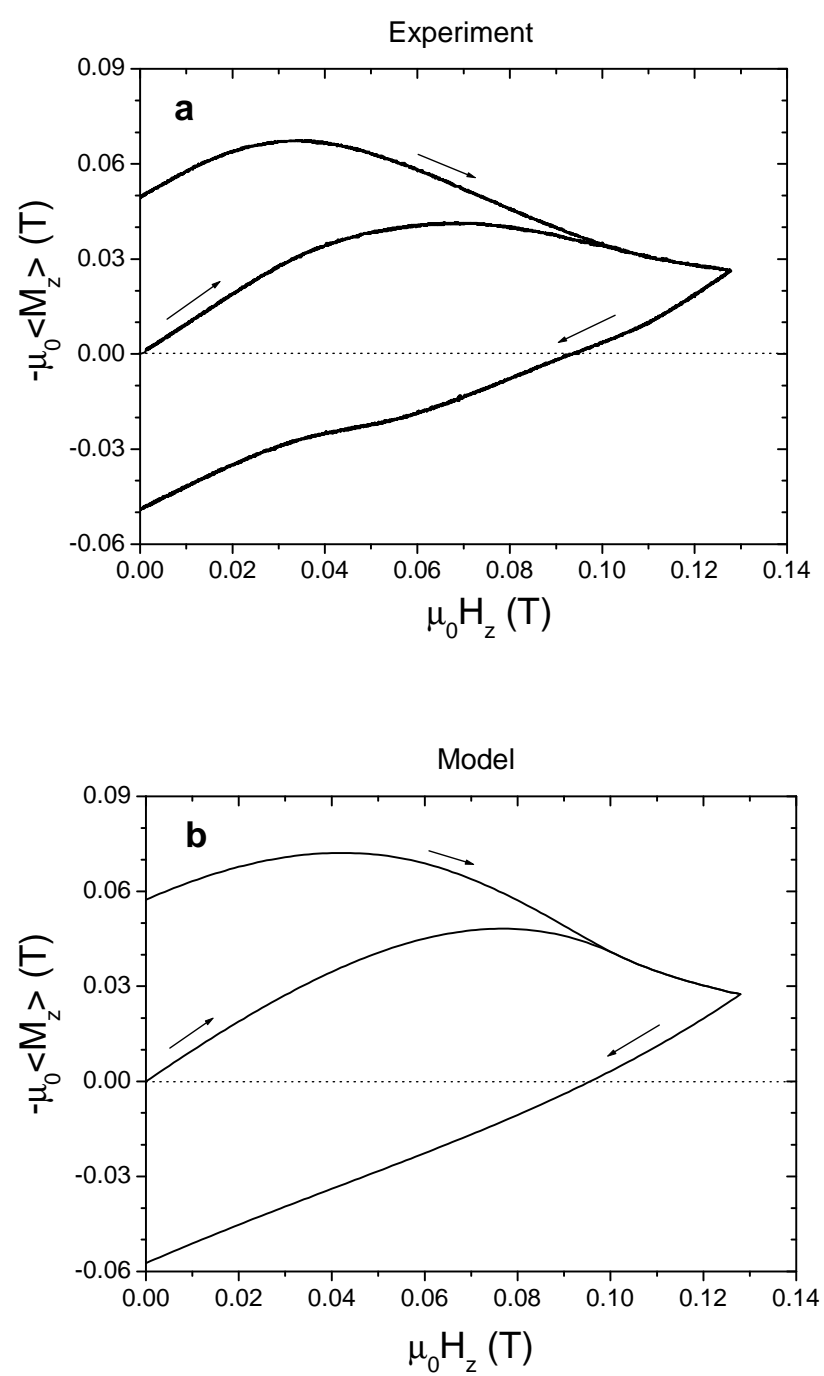

Figure 1: Standard magnetization curves (a) for a PbBi disk, taken from Ref. 33. Theoretical magnetization curves (b) obtained with a critical current density $J_{c \perp}(B)$ as in Eq. 3.31.

As it is seen in Fig. 2, for the smallest value of $H_{a}$ $\left(=0.5 H_{P}\right.$, panel a), both magnetization components have a nonmonotonic behavior as functions of $\theta$. Such a behavior of magnetization has also been observed in Ref. [11] during the initial rotation of a Nb disk undergoing slow oscillations in a parallel field. The dependence of the magnetization on $\theta$ radically changes at larger values of $H_{a}$. So (see Fig. 2, b), at $H_{a}=H_{P}$ the functions $\left\langle M_{y}>(\theta)\right.$ and $-<M_{z}>(\theta)$ initially grow with $\theta$ and later (at $\theta>150^{\circ}$ ) they practically become constants with close values $\left.\left(<M_{y}>\approx-<M_{z}\right\rangle\right)$. Also note that $M_{y}$ has a maximum at $\theta \approx 75^{\circ}$. For $H_{a}$ larger than the penetration field $H_{P}$ (panel c), the function $-<M_{z}>(\theta)$ takes values smaller than those for $\left\langle M_{y}>(\theta)\right.$. Both of them are almost constant functions, except at small rotation angles because of 

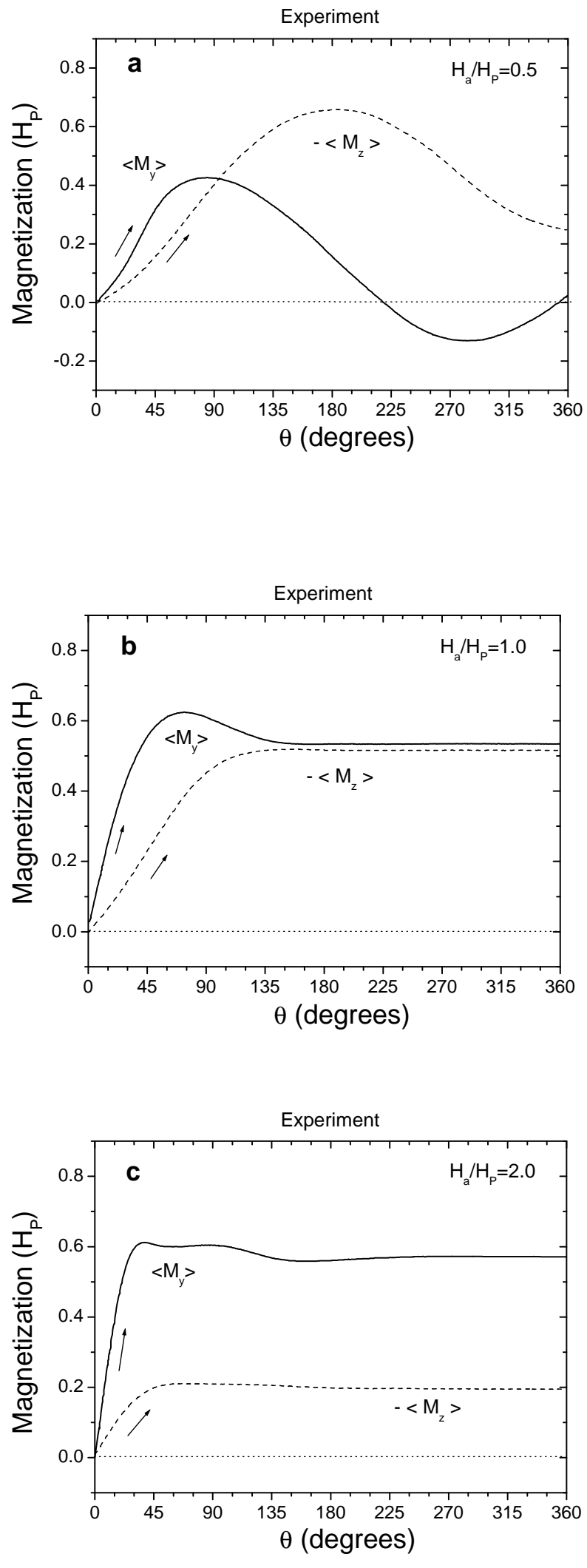

Figure 2: PbBi rotational curves measured in Ref. [33]. their fast initial growth. Thus, the maximum of $<$ $M_{y}>$ is shifted to a smaller value of $\theta\left(\approx 40^{\circ}\right)$.

\section{B. Theoretical predictions}

The models described in the previous section can be applied to explain the experimental results (Fig. 22 if we fix the sample and rotate the external magnetic field $\boldsymbol{H}_{a}$ (2.1) by an angle $\alpha_{s}=-\theta$ instead of fixing the magnetic field and rotating the superconducting sample. Then, the experimental values $\left\langle M_{y}\right\rangle$ and $\left.-<M_{z}\right\rangle$ should respectively correspond to the quantities:

$$
\begin{array}{r}
<M_{y}>=\frac{1}{\mu_{0} d} \int_{0}^{d} d x B_{y}^{\prime}(x), \\
-<M_{z}>=H_{a}-\frac{1}{\mu_{0} d} \int_{0}^{d} d x B_{z}^{\prime}(x),
\end{array}
$$

where

$$
\begin{gathered}
B_{y}^{\prime}=\hat{\boldsymbol{\alpha}}_{s} \times \hat{\boldsymbol{x}} \cdot \boldsymbol{B}=B(x) \sin \left[\alpha(x)-\alpha_{s}\right], \\
B_{z}^{\prime}=\hat{\boldsymbol{\alpha}}_{s} \cdot \boldsymbol{B}=B(x) \cos \left[\alpha(x)-\alpha_{s}\right] .
\end{gathered}
$$

The calculations of magnetization components < $M_{y}>$ and $-<M_{z}>$ with the critical-state models, discussed in Sec. II. require the employment of the parameters $J_{c \perp}(B)$ and $J_{c \|}(B)$, depending on the magnetic induction. The former, $J_{c \perp}(B)$, is determined from the experimental curves of magnetization versus the applied field, varying along one direction only as in Fig. 1 (In this case, flux cutting does not occur and, consequently, the depinning effects are completely responsible for the magnetic response of the superconductor). The standard magnetization curves are well reproduced by any one of the critical-state models (see above) with

$$
J_{c \perp}(B)=\frac{J_{c \perp}(0)}{\left(1+B / \mu_{0} H_{P}\right)^{n_{\perp}}}
$$

$J_{c \perp}(0)=47.11 \times 10^{7} \mathrm{~A} / \mathrm{m}^{2}$, and $n_{\perp}=2$ (compare panels (a) and (b) of Fig. 1). Other parameters of the critical state models are found by adjusting theoretical magnetization curves to the experimental ones (Fig. 2 .

\section{Circular model}

Within the Bean's circular critical-state model (2.11), there is only one phenomenological parameter, i.e. $J_{c}(B)=J_{c \perp}(B)=J_{c \|}(B)$. Then, $J_{c}(B)$ has the form (3.31) with the same values for the parameters $J_{c \perp}(0)$, and $n_{\perp}$.

Fig. 3 shows our numerical results for $\left\langle M_{y}\right\rangle$ and $-<M_{z}>$, obtained with the Bean critical-state model. At first glance, it seems that the circular model qualitatively reproduces the experimental magnetization curves (Fig. 2). However, there are important differences between its predictions and the experiment. 

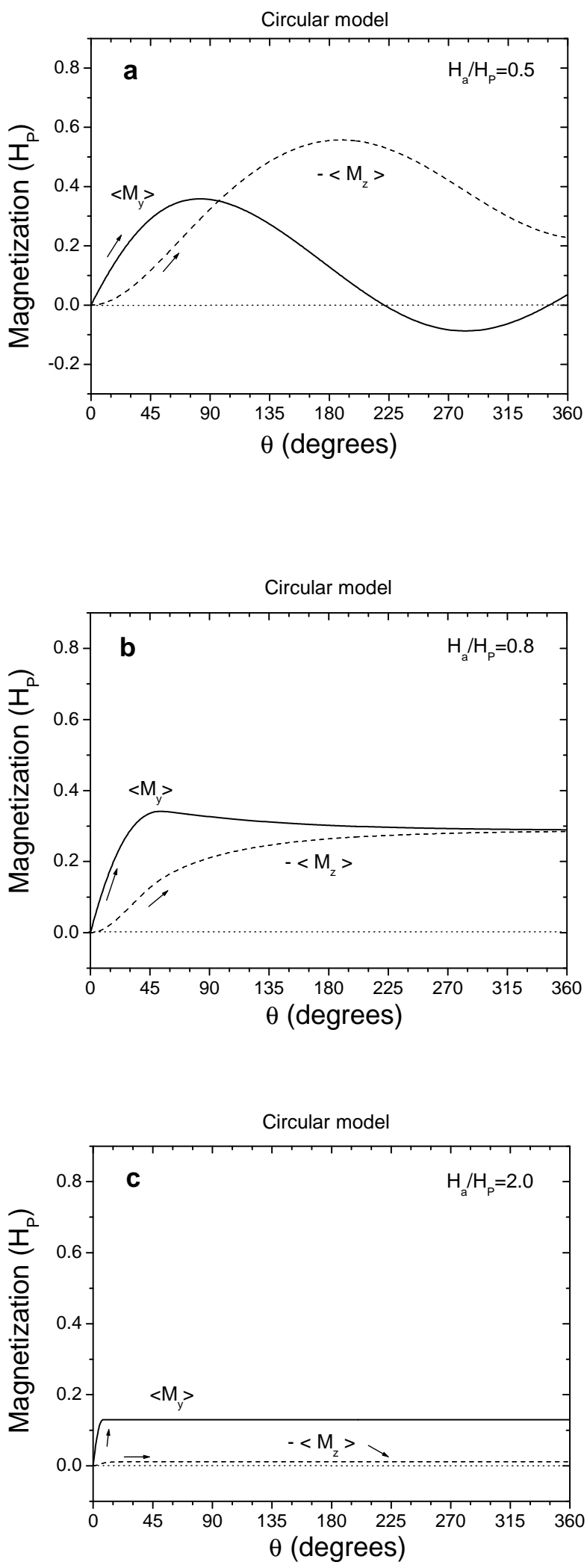

Figure 3: Curves of the average magnetization components versus the rotation angle, calculated with Bean's critical-state model.
Thus, for example, the "oscillations" of the magnetization components (Fig. 3 a) have small amplitudes compared with the experimental ones. Besides, at $H_{a}=$ $0.8 H_{P}$ the functions $\left\langle M_{y}>(\theta)\right.$ and $-<M_{z}>(\theta)$ approximate each other but at relatively large rotation angles $\theta>300^{\circ}$. Finally, when the applied field has an amplitude larger than $H_{p}$ (see panel c), the magnetization components are rather small in magnitude and their initial growth, before the saturation, occurs in a very small interval of $\theta\left(<20^{\circ}\right)$.

\section{Elliptic model}

The calculations of magnetization components $\left\langle M_{y}\right\rangle$ and $-<M_{z}>$ within the elliptic flux-line-cutting critical-state model (2.14) are shown in Fig. 4. Here, we used the same $J_{c \perp}(B)$ as in Eq. (3.31) and $J_{c \|}(B)$ of the form

$$
J_{c \|}(B)=\frac{J_{c \|}(0)}{\left(1+B / \mu_{0} H_{P}\right)^{n_{\|}}}
$$

with $J_{c \|}(0)=1.5 J_{c \perp}(0)$ and $n_{\|}=1$. This choice provides a good agreement between experimental (Fig. 2) and theoretical (Fig. 4) curves. Thanks to the use of a second parameter $\left(J_{c \|}\right)$, the elliptic model is able to generate the "oscillations" of the magnetization components (Fig. 4,a) with amplitude close to that observed in the experiment (panel (a) in Fig. 2). Notice that $\left\langle M_{y}>\right.$ and $-<M_{z}>$ approach each other at $\theta>150^{\circ}$ with $H_{0}=1.05 H_{P}$ in good concordance with the measurements (see Fig. 2,b, corresponding to $H_{0}=H_{P}$ ). In addition, when $H_{0}=2.0 H_{p}$ (panel (c) in Fig. 4), the difference between $\left\langle M_{y}\right\rangle$ and $-\left\langle M_{z}\right\rangle$ at $\theta>45^{\circ}$ is as large as in the experiment (Fig. 4,c).

\section{Extended elliptic model}

As was commented in Sec. II. both elliptic and circular critical-state models are particular cases of the extended elliptic one. Therefore, the results presented in Fig. 3, predicted by the circular model, can also be calculated by using the new model (Eqs. 2.21) and (2.22) with $J_{c \perp}=J_{c \|}$ as in Eq. (3.31 and $r=\rho_{\|} / \rho_{\perp}=\rho_{c} / \rho_{d}$ being equal to one $(r=1)$ at $J>J_{c}$. The condition $r=1$ guarantees that the electric field $\boldsymbol{E}$ and current density $\boldsymbol{J}$ be parallel as it is postulated by Bean's critical-state model (2.11). In addition, graphs in Fig. 4 (original elliptic model predictions), which quantitatively reproduce experimental measurements (Fig. 2), are also obtained with the extended elliptic critical-state model (Eqs. 22.21) and (2.22) ) if $r=J_{c \perp} / J_{c \|}$ (i.e. $\left.\rho_{c} / \rho_{d}=J_{c \perp} / J_{c \|}\right)$. According to the parameters $J_{c \perp}(B) \sqrt{3.31}$ and $J_{c \|}(B)$ (3.32), used for calculating magnetization curves in Fig. 4, the ratio $r$ is here smaller than $1(r<1)$.

It is interesting to study the effect of the parameter $r$, controlling the relation between the electric field $\boldsymbol{E}$ 

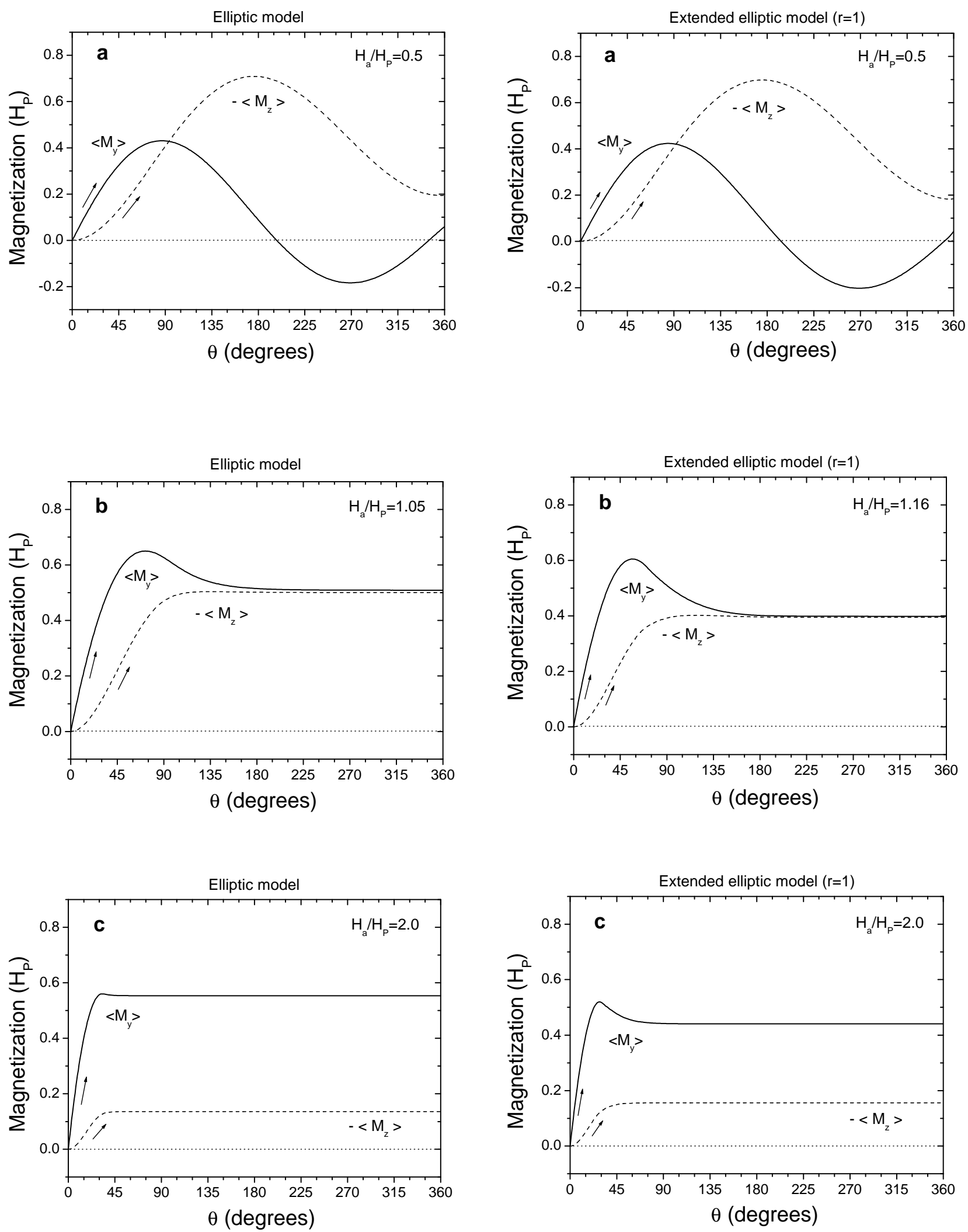

Figure 4: Curves of the average magnetization components versus the rotation angle, calculated with the original elliptic critical-state model.

Figure 5: Curves of the average magnetization components versus the rotation angle, calculated with the extended elliptic critical-state model using a ratio $r=1$. 

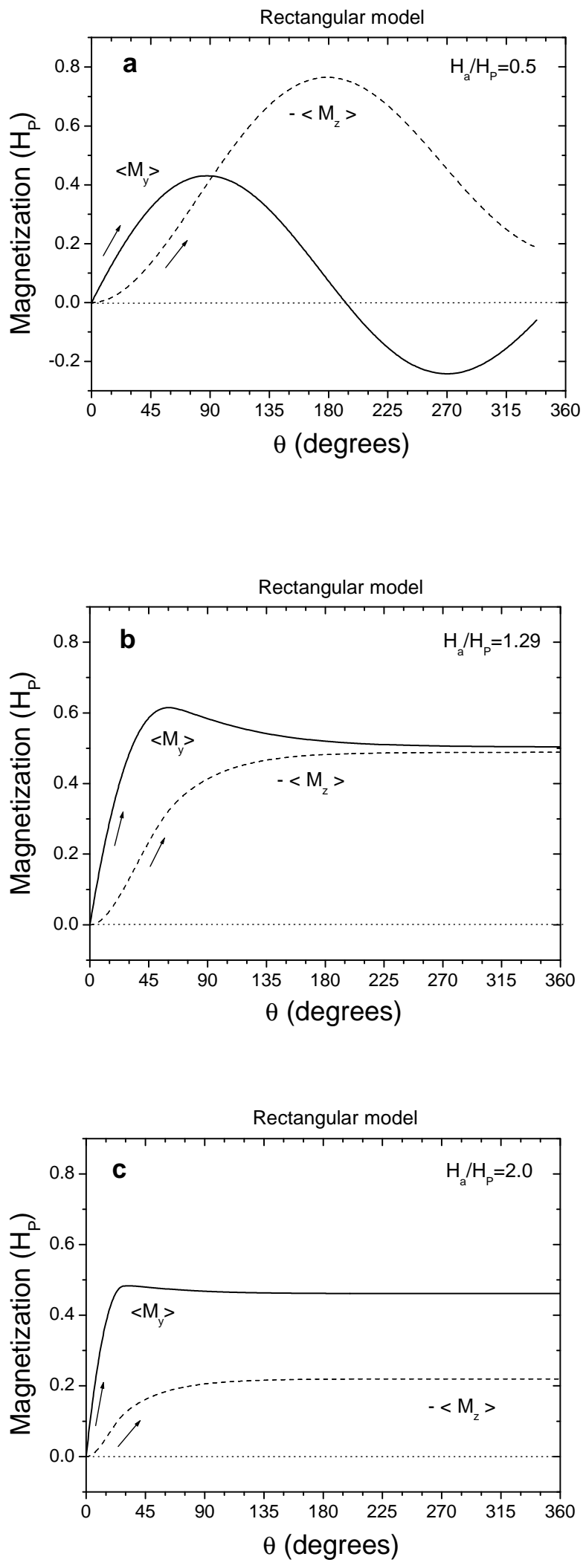

Figure 6: Curves of the average magnetization components versus the rotation angle, calculated with the generalized double critical-state model. and the current density $\boldsymbol{J}$ at $J>J_{c}$. For this reason, we have calculated magnetization curves (Fig. 5) by applying the extended elliptic model with the same parameters $J_{c \perp}(B)$ and $J_{c \|}(B)$ as those employed in Fig. 4. but with the parameter $r=\rho_{c} / \rho_{d}=1$. In other words, the magnetization curves in Fig. 5 correspond to an anisotropic critical-state model with $J_{c \perp} / J_{c \|}<1$, but the parameter $r=1$, indicating that $\boldsymbol{E}$ and $\boldsymbol{J}$ are parallel when $J>J_{c}$. From the comparison of Fig. 5 with 4 we note that magnetization curves significantly depend upon the parameter $r$ when the applied magnetic field is large enough $\left(H_{a}>H_{P}\right.$ as in panels (b) and (c)). So, in order the magnetization components, $\left\langle M_{y}>\right.$ and $-\left\langle M_{z}>\right.$, to have the same value at large angles of rotation, the applied magnetic field $H_{a}$ for $r=1$ (Fig. 5 b) should be larger than the field used in Fig. 4. b. Besides, the value of $\left\langle M_{y}\right\rangle$ and $\left.-\left\langle M_{z}\right\rangle \approx 0.4 H_{P}\right)$, at sufficiently large angles $\theta$, turns out to be smaller than that $\left(\approx 0.5 H_{P}\right)$ predicted by the original elliptic model (Fig. 4 4 b). At $H_{a}=2.0 H_{P}$, there is also a noticeable difference between magnetization $y$-components (compare panels (c) of Figs. 4 and 5 .

\section{Rectangular model}

For completeness of our study, we have employed the GDCSM (rectangular model), which also uses two critical current densities, namely $J_{c \perp}(B)$ and $J_{c \|}(B)$. The former is determined from the curves of magnetization versus the applied field, varying along one direction only (Fig. 1). In our case, the magnetic dependence of $J_{c \perp}$ is the same as in Eq. (3.31). To reproduce the main features of the experiment (Fig. 2), the other parameter is chosen as in Eq. (3.32), but $J_{c \|}(0)=1.32 J_{c \perp}(0)$ and $n_{\|}=1.06$ (compare Figs. 2 and 6). Although these values are different from those used within the elliptic critical-state model, the parallel critical current density $J_{c \|}$ remains being larger than the perpendicular one $J_{c \perp}$. It should be noted that the GDCSM predicts the equality of $\left\langle M_{y}>\right.$ and $-\left\langle M_{z}>\left(\approx 0.5 H_{P}\right)\right.$ with an external field $H_{a}=1.29 H_{P}>H_{P}$ at relatively large rotation angles $\theta>270^{\circ}$ (see Fig. 6 b), in contrast to the experiment where such a behavior occurs from $\theta \approx 150^{\circ}$. Besides, the numerical calculations for $H_{a}=0.5 H_{P}$ (panel (a) in Fig. 6) had to be stopped at $\theta \approx 338^{\circ}$ because the solution further diverged.

\section{Magnetic induction profiles}

The fact that the elliptic critical-state model is able to quantitatively reproduce the experiment, with the use of a parallel critical current density $J_{c \|}(B)$ larger than the perpendicular one $J_{c \perp}(B)$, illustrates how flux-line cutting influences on the magnetic behavior of a rotating superconductor. To explain the features observed in both experimental (Fig. 2) and theoretical (Fig. 4) magnetization curves, we shall analyze the evolution 

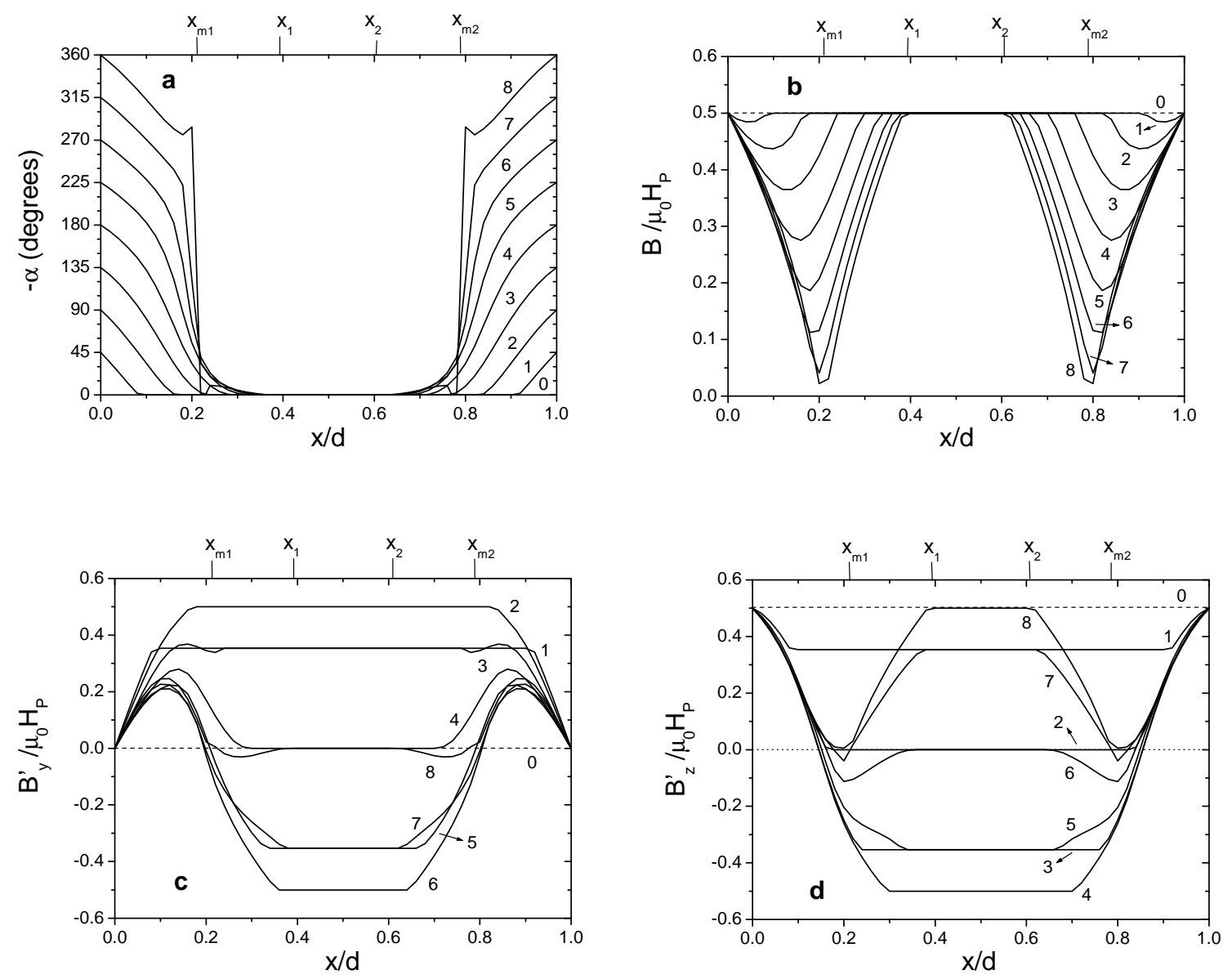

Figure 7: Profiles of the angle $\alpha$ (panel a), magnitude $B$ (panel b) and components $B_{y}^{\prime}$ (Eq. 3.29), panel c) and $B_{z}^{\prime}$ (Eq. (3.30), panel d) of the magnetic induction, calculated with the original elliptic critical-state model at $H_{a}=0.5 H_{P}$.

of the profiles for the magnitude of the magnetic induction $B(x)$, the tilt angle $\alpha(x)$, and the components $B_{y}^{\prime}(x) \sqrt{3.29}$ and $B_{z}^{\prime}(x) 3.30$, calculated within the original elliptic flux-line-cutting critical-state model (Figs. 7, 9).

The calculated profiles of the magnetic induction in the case when the external magnetic field $H_{a}$ has a magnitude smaller than the penetration field $H_{p}$ $\left(H_{a}=0.5 H_{p}\right)$ are shown in Fig. 7. As the angle of rotation is increased, two $U$-shaped minima in the $B(x)$ profile (panel b) appear because of the flux consumption (decrement of $B$ ) which results from flux-line cutting 4. The absolute value of the tilt angle $\alpha$ increases with $\theta$ in the near-surface intervals $0 \leq x<x_{m 1}$ and $x_{m 2}<x \leq d$. However, in the intervals $x_{m 1}<x<x_{1}$ and $x_{2}<x<x_{m 2}$, where there is flux consumption, the angle $\alpha$ is slightly modified. In the central interval, $x_{1}<x<x_{2}$, neither $B$ or $\alpha$ is altered. When $\theta \approx 360^{\circ}$, the minimum values of $B$ inside the superconducting disk tend to zero and, as follows from Eq. 2.8, the magnitude of the derivative $\partial \alpha / \partial x$ considerably increases at such points. Besides, at $x=x_{m 1}$ and $x=x_{m 2}$ with $B\left(x_{m 1}\right)=B\left(x_{m 2}\right) \approx 0$, the accuracy of our calculations is low and, therefore, the values $-\alpha\left(x_{m 1}\right)$ and $-\alpha\left(x_{m 2}\right)$ turned out to be apparently higher than they should be (see curve 8 for $\theta=360^{\circ}$ in Fig. 7 ,a). The component $B_{z}^{\prime}$ of the magnetic induction, parallel to the applied magnetic field $\mathbf{H}_{a}$, decreases near sample surfaces because of the flux consumption (Fig. 7,d). Nevertheless, the most important change occurs in the central part of the sample (in $x_{1}<x<x_{2}$ ) because of the sample rotation. So, at $\theta=180^{\circ}$ (curve 4) the component $B_{z}^{\prime}$ varies from $B_{z}^{\prime}=\mu_{0} H_{a}$ at the surfaces $x=0$ and $x=d$ to the opposite value $B_{z}^{\prime}=-\mu_{0} H_{a}$ in the central region of the sample. When an entire cycle is finished, $B_{z}^{\prime}$ again takes the value $B_{z}^{\prime}=\mu_{0} H_{a}$ in the middle of the disk (curve 8). This cyclic behavior of $B_{z}^{\prime}$ is responsible for the "oscillations" of the magnetization component $<M_{z}>(\theta)$ (panels (a) in Figs. 2 and 4), being negative for any value of the angle of rotation $\theta>0$ because $B_{z}^{\prime}<\mu_{0} H_{a}$ near surfaces, i.e. in the intervals $0<x<x_{1}$ and $x_{2}<x<d$. The component $B_{y}^{\prime}$ also oscillates in the middle of the sample as $\theta$ is increased 

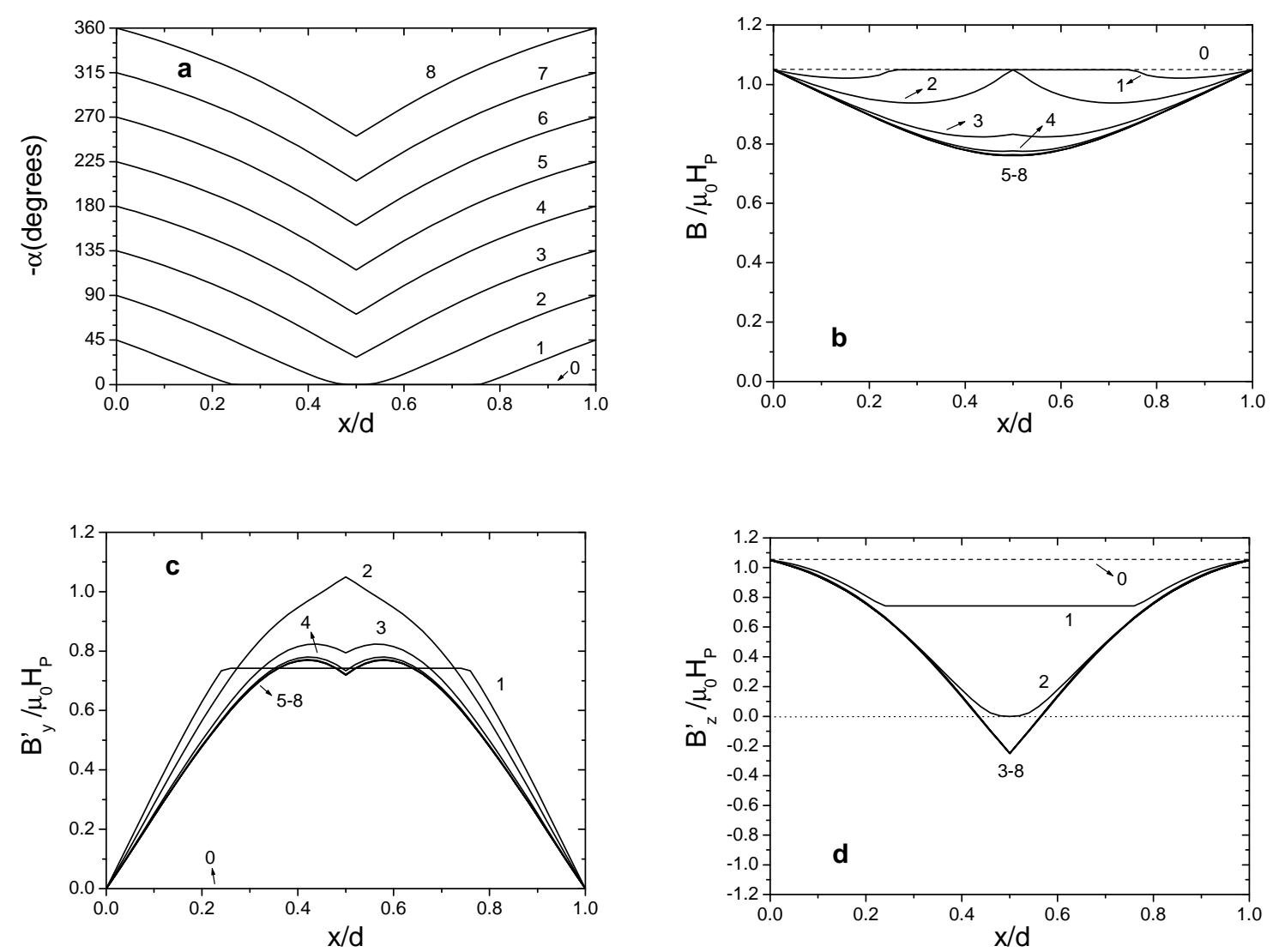

Figure 8: Profiles of the angle $\alpha$ (panel a), magnitude $B$ (panel b) and components $B_{y}^{\prime}$ (Eq. 3.29), panel c) and $B_{z}^{\prime}$ (Eq. (3.30), panel d) of the magnetic induction, calculated with the original elliptic critical-state model at $H_{a}=1.05 H_{P}$.

(Fig. 7,c). Such a behavior of $B_{y}^{\prime}$ makes the magnetization $y$-component $\left\langle M_{y}\right\rangle$ oscillate with $\theta$ (Figs. 2 , a and 4 a). As it is seen in Fig. 7k, there is an increment of $B_{y}^{\prime}$ in the near-surface regions, producing a small positive value for $\left\langle M_{y}\right\rangle(3.27)$ after a complete cycle, i.e. at $\theta=360^{\circ}$ (see Figs. 2 a and 4,a).

Fig. 8 exhibits profiles calculated within the elliptic critical-state model for $H_{a}=1.05 H_{p}$. Due to the decrease of the critical current densities $J_{c \perp}(3.31)$ and $J_{c \|} \sqrt{3.32}$ with the magnitude $B$ of the magnetic induction, the slopes of the critical profiles for $B(x)$ and $\alpha(x)$ near surfaces are smaller than the slopes observed in the corresponding profiles of Fig. 7. Therefore, the central region with unaltered $B$ and $\alpha$ (see curves 1 in panels (a) and (b) of Fig. 8 rapidly disappears as the rotation angle $\theta$ is increased (see curves 2 therein). Also, the $U$-shaped minima of $B(x)$ coalesce forming a unique minimum at the center of the disk. The resulting critical profile $B(x)$ does not further change despite the fact that the disk continues rotating (see curves 58 in panel $(\mathrm{b}))$. In this case, $B_{z}^{\prime}(x)$ initially decreases (curves 1-2 in Fig. 8,d) inside the sample as $\theta$ varies until it reaches the critical profile (curves 3-8). Hence, the dependence $\left\langle M_{z}\right\rangle(\theta)$ has a monotonic behavior at $\theta>120^{\circ}$ (see panels (b) in Figs. 2 and 4 ). On the other hand, $B_{y}^{\prime}(x)$ increases so that a huge maximum in the dependence $\left\langle M_{y}\right\rangle(\theta)$ (Figs. 2,b and 4,b) appears at $\theta \approx 70^{\circ}$. At large rotation angles $\left(\theta>180^{\circ}\right)$, the profile $B_{y}^{\prime}(x)$ becomes stationary and $\left\langle M_{y}\right\rangle(\theta)$ is, practically, a constant function, having a value close to $-\left\langle M_{z}\right\rangle$. So, the magnitude of the magnetization, $|<\mathbf{M}>|$, is independent of $\theta$ when the rotation angle is sufficiently large.

The profiles for the case when the external magnetic field is large enough, in comparison with the penetration field $H_{P}$ (as in Fig. 9), have an evolution similar to that presented in Fig. 8. However, the central regions of unaltered magnetic induction rapidly disappear as $\theta$ is increased, in comparison with the results of Fig. 8 This fact is due to noticeable reduction of the critical current densities $J_{c \perp}$ and $J_{c \|}$ with $B$.

\section{CONCLUSION}

We have applied the circular, elliptic, extended-elliptic, and rectangular critical-state models to study the magnetic behavior of irreversible type-II superconductors 

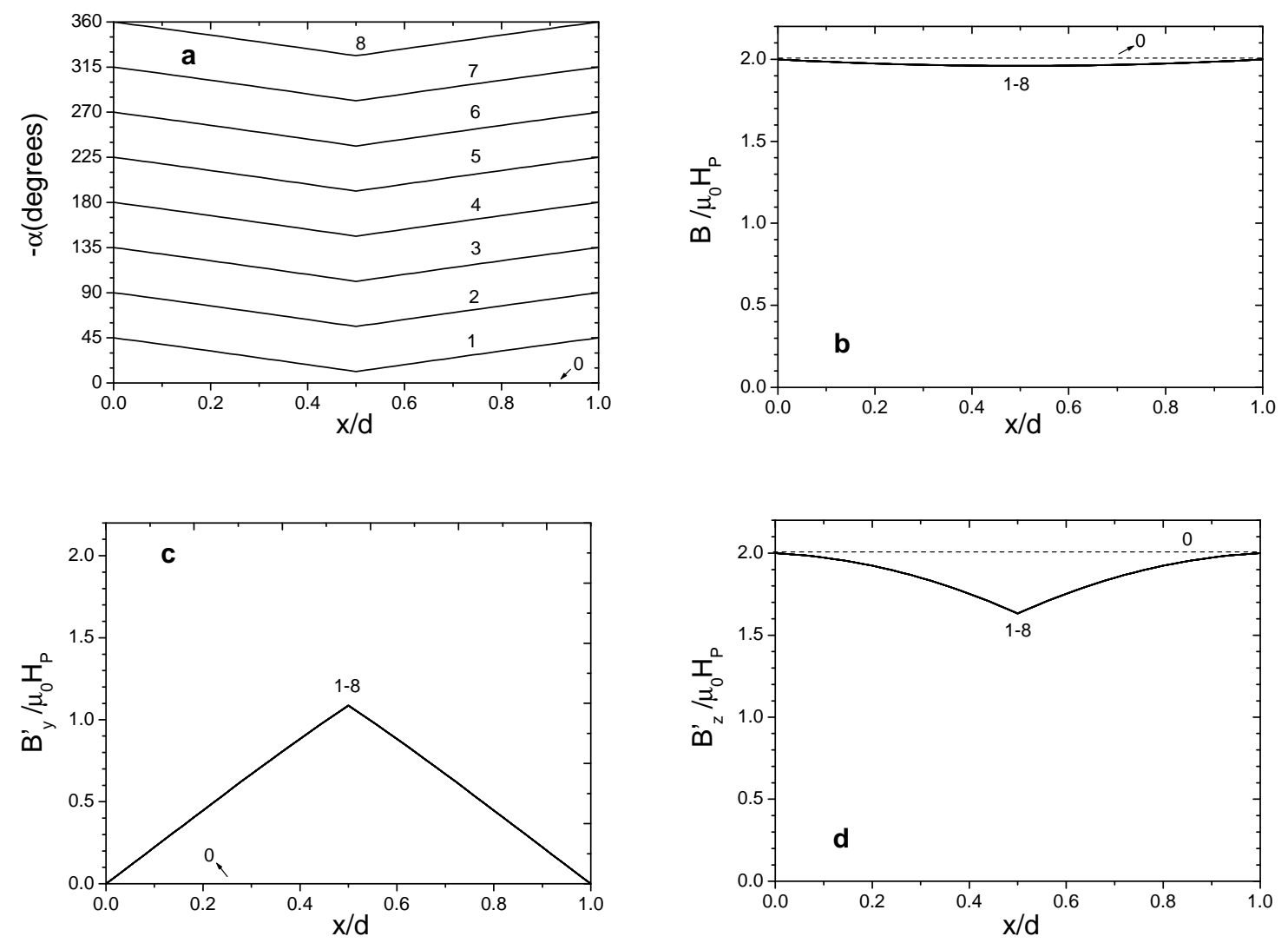

Figure 9: Profiles of the angle $\alpha$ (panel a), magnitude $B$ (panel b) and components $B_{y}^{\prime}$ (Eq. 3.29 , panel c) and $B_{z}^{\prime}$ (Eq. (3.30), panel d) of the magnetic induction, calculated with the original elliptic critical-state model at $H_{a}=2.0 H_{P}$.

in a parallel rotating magnetic field. The numerical method employed here is based on the substitution of the vertical law, relating the electric field $\boldsymbol{E}$ and the current density $\boldsymbol{J}$, for a nonlinear material equation having effective flux-cutting and flux-flow resistivities in the dissipative region. The substitution is justified when the applied magnetic field $H_{a}$ slowly varies either in magnitude or direction, inducing electric fields of sufficiently small magnitude inside the superconductor. Within the elliptic (circular) critical-state model such resistivities are not independent of each other and have a ratio $r=\rho_{\|} / \rho_{\perp}$ equal to $J_{c \perp} / J_{c \|}(=1$ for the circular model) at $J$ just above its critical value $J_{c}$. On the other hand, within the extended elliptic critical-state model the ratio $r$ is an independent parameter to be determined. The rectangular critical-state model also uses two independent resistivities, $\rho_{\|}$and $\rho_{\perp}$. However, unlike the other critical-state models, the GDCSM assumes that flux cutting and flux depinning do not affect each other.

The comparison of the predictions of the mentioned critical-state models with experimental measurements of magnetization for a rotating $\mathrm{PbBi}$ disk in a fixed magnetic field 33 shows that the original critical-state model can reproduce the main features of the magne- tization curves. The circular and rectangular criticalstate models only achieve a qualitative description of the experiment. The extended elliptic model, being more general than the original elliptic one, has allowed us to study the effect of the relation between $\boldsymbol{E}$ and $\boldsymbol{J}$ in the dissipative region. However, additional theoretical and experimental studies are needed to elucidate on the effects associated with both flux-cutting and flux-flow resistivities.

\section{Acknowledgements}

This work was partially supported by Consejo Nacional de Ciencia y Tecnología (CONACYT, Mexico).

\section{References}

[1] L. M. Fisher, A. V. Kalinov, I. F. Voloshin, I. V. Baltaga, K. V. Il'enko, V. A. Yampol'skii, Solid State Commun. 97, 833 (1996).

[2] C. P. Bean, Phys. Rev. Lett. 8, 250 (1962).

[3] C. P. Bean, J. Appl. Phys. 41, 2482 (1970).

[4] J. R. Clem, Phys. Rev. B 26, 2463 (1982). 
[5] J. R. Clem and A. Pérez-González, Phys. Rev. B 30, 5041 (1984).

[6] A. Pérez-González and J. R. Clem, Phys. Rev. B 31, 7048 (1985).

[7] A. Pérez-González and J. R. Clem, Phys. Rev. B 32, 2909 (1985).

[8] A. Pérez-González and J. R. Clem, J. Appl. Phys. 58, 4326 (1985).

[9] D. G. Walmsley, J. Phys. F 2, 510 (1972).

[10] A. M. Campbell and J. E. Evetts, Adv. Phys. 21, 199 (1972).

[11] J. R. Cave and M. A. R. LeBlanc, J. Appl. Phys. 53, 1631 (1982).

[12] R. Boyer and M. A. R. LeBlanc, Solid State Commun. 24, 261 (1977).

[13] R. Boyer, G. Fillion, and M. A. R. LeBlanc, J. Appl. Phys. 51, 1692 (1980).

[14] M. A. R. LeBlanc and J. P. Lorrain, J. Appl. Phys. 55, 4035 (1984).

[15] F. Pérez-Rodríguez, A. Pérez-González, J. R. Clem, G. Gandolfini, and M. A. R. LeBlanc, Phys. Rev. B 56, 3473 (1997).

[16] A. Silva-Castillo, R. A. Brito-Orta, A. PérezGonzález, and F. Pérez-Rodríguez, Physica C 296, 75 (1998).

[17] L. M. Fisher, K. V. Il'enko, A. V. Kalinov, M. A. R. LeBlanc, F. Pérez-Rodríguez, S. E. Savel'ev, I. F. Voloshin, V. A. Yampol'skii, Phys. Rev. B 61, 15382 (2000).

[18] I. F. Voloshin, L. M. Fisher, V. A. Yampol'skii, Low Temp. Phys. 36, 39 (2010).

[19] C. Romero-Salazar and F. Pérez-Rodríguez, Appl. Phys. Lett. 83, 5256 (2003).
[20] I. F. Voloshin, A. V. Kalinov, L. M. Fisher, A. V. Aksenov, and V. A. Yampol'skii, JETP 93, 1105 (2001).

[21] C. Romero-Salazar and F. Pérez-Rodríguez, Supercond. Sci. Technol. 16, 1273 (2003).

[22] C. Romero-Salazar and F. Pérez-Rodríguez, Physica C 404, 317 (2004).

[23] L. M. Fisher, A. V. Kalinov, S. E. Savelev, I. F. Voloshin, V. A. Yampol'skii, M. A. R. LeBlanc, and S. Hirscher, Physica C 278, 169 (1997).

[24] L. M. Fisher, A. V. Kalinov, S. E. Savelev, I. F. Voloshin, and V. A. Yampol'skii, Solid State Commun. 103, 313 (1997).

[25] C. Romero-Salazar, L. D. Valenzuela-Alacio, A. F. Carballo-Sánchez, and F. Pérez-Rodríguez, J. Low Temp. Phys. 139, 273 (2005).

[26] J. P. Lorrain, M. A. R. LeBlanc, and A. Lachaine, Can. J. Phys. 57, 1458 (1979).

[27] C. Romero-Salazar and O. A. Hernández-Flores, J. Appl. Phys. 103, 093907 (2008).

[28] J. R. Clem, M. Weigand, J. H. Durrell, and A. M. Campbell, arXiv:1103.1393v1 [condmat.supr-con] 7 Mar 2011.

[29] E. H. Brandt and G. P. Mikitik, Phys. Rev. B 76, 064526 (2007).

[30] G. P. Mikitik, Low Temp. Phys. 36, 13 (2010).

[31] J. R. Clem, arXiv:1102.3678v1 [cond-mat.suprcon] 17 Feb 2011.

[32] A. Badía-Majós, C. López, and H. S. Ruiz, Phys. Rev. B 80, 144509 (2009).

[33] J. Sekerka, M.Sc. thesis "Flux cutting in semireversible and irreversible type II superconductors", University of Ottawa, 1989.

[34] C. Romero-Salazar and F. Pérez-Rodríguez, J. Non-Cryst. Sol. 329, 159 (2003). 